\title{
Paléolacs et peuplements holocènes du Yémen : le Ramlat As-
} Sabat'ayn.

Marie-Louise Inizan, Anne-Marie Lezine, Bruno Marcolongo, Jean-François Saliège, Christian Robert, Frédéric Werth

\section{Citer ce document / Cite this document :}

Inizan Marie-Louise, Lezine Anne-Marie, Marcolongo Bruno, Saliège Jean-François, Robert Christian, Werth Frédéric. Paléolacs et peuplements holocènes du Yémen : le Ramlat As-Sabat'ayn.. In: Paléorient, 1997, vol. $23, \mathrm{n}^{\circ} 2$. Paléoenvironnement et sociétés humaines au moyen-orient de 20000 BP à 6 000 BP. pp. 137-149;

doi : https://doi.org/10.3406/paleo.1997.4657

https://www.persee.fr/doc/paleo_0153-9345_1997_num_23_2_4657

Fichier pdf généré le 24/04/2018 


\begin{abstract}
The surveys in the Ramlat as-Sabat'ayn started in 1983 and several prehistoric periods have been recognized from the Lower Palaeolithic up to the Bronze Age according to the lithic industry and to many structures. Only one period could accurately be dated about 7,500 BP through the discovery of a paleolake, which corresponds to the early Holocene and which is related to the period of climatic improvement resulting from the intensification of the Indian monsoon. The filling up of this lake starts as early as $8,700 \mathrm{BP}$. The vegetal environment corresponds to steppic conditions which have lasted while preserving an important evaporation. The first observations concerning the lithic industry show typological and technological affinities (grinding stones, mortars, pestles, stones vessels, projectile points, arrow-heads, pressure flaking, etc.) with the Rub'al-Khali neolithic industries.
\end{abstract}

\title{
Résumé
}

Les prospections dans le Ramlat as-Sabat'ayn ont débuté en 1983 et plusieurs périodes d'occupations préhistoriques ont été reconnues depuis le Paléolithique ancien jusqu 'à I 'âge du Bronze grâce à l'industrie lithique et à de nombreuses structures d'habitat et funéraires. La seule période qui a pu être datée avec précision, aux alentours de 7500 BP grâce à la découverte d'un lac fossile, concerne l'Holocène ancien correspondant à la période d'amélioration climatique liée à i intensification de la mousson indienne. Le remplissage de ce lac débute dès $8700 \mathrm{BP}$. L'environnement végétal correspond à des conditions steppiques qui ont perduré, maintenant une importante evaporation. Les premières observations sur l'industrie lithique de cette région indiquent des affinités typologiques et technologiques (armatures de jet, matériel de broyage, vaisselle de pierre, présence de la retouche par pression en écharpe) avec des industries néolithiques du Rub'al-Khali. 


\title{
PALÉOLACS ET PEUPLEMENTS HOLOCÈNES DU YÉMEN : LE RAMLAT AS-SABAT'AYN
}

\author{
M.-L. INIZAN, A.-M. LEZINE, B. MARCOlONGO, J.-F. SALIEGE, C. ROBERT et F. WERTH
}

\begin{abstract}
Résumé : Les prospections dans le Ramlat as-Sabat'avn ont débuté en 1983 et plusieurs périodes d'occupations préhistoriques ont été reconnues depuis le Paléolithique ancien jusqu'à l'âge du Bronze grâce à l'industrie lithique et à de nombreuses structures d'habitat et funéraires. La seule période qui a pu être datée avec précision, aux alentours de 7500 BP grâce à la découverte d'un lac fossile, concerne l'Holocène ancien correspondant à la période d'amélioration climatique liée à l'intensification de la mousson indienne. Le remplissage de ce lac débute dès 8700 BP. L'environnement végétal correspond à des conditions steppiques qui ont perduré, maintenant une importante évaporation. Les premières observations sur l'industrie lithique de cette région indiquent des affinités typologiques et technologiques (armatures de jet, matériel de brovage, vaisselle de pierre, présence de la retouche par pression en écharpe) avec des industries néolithiques du Rub'al-Khali.
\end{abstract}

Abstract: The suneys in the Ramlat as-Sabat'ayn started in 1983 and several prehistoric periods have been recognized from the Lower Palaeolithic up to the Bronse Age according to the lithic industry and to many structures. Only one period could accurately. be dated about 7,500 BP through the discovery of a paleolake, which corresponds to the early Holocene and which is related to the period of climatic improvement resulting from the intensification of the Indian monsoon. The filling up of this lake starts as early as 8,700 BP. The vegetal environment corresponds to steppic conditions which have lasted while preserving an impontant evaporation. The first obsenations concerning the lithic industry show typological and technological affinities (grinding stomes, mortars, pestles, stones vessels, projectile points, arrow-heads, pressure flaking, etc.) with the Rub'al-Khali neolithic industries.

Mots clefs : Yémen, Arabie, Holocène, Paléolac, Néolithique, Pollen, Isotopes stables, Sédiments, Minéralogie des argiles.

Key-words: Yemen, Arabia, Holocene, paleolake, Neolithic, Pollen, Stable isotope, Sediment, Clay minerals.

\section{INTRODUCTION}

La première reconnaissance des sites préhistoriques dans le bassin désertique du Ramlat as-Sab'atayn et ses bordures a commencé en 1983-1984 dans la région du site antique de Shabwa (fig. 1). Elle s'inscrivait dans la recherche du processus du peuplement de cette région qui a vu le développement de la civilisation sudarabique. En effet, c'est dans ce bassin que viennent se perdre, lors des crues, les eaux des wâdîs le long desquels se sont installés les royaumes agricoles antiques, grâce surtout à l'exploitation de systèmes d'irrigation très complexes!.

1. BRIT(N. 1992.
Dès 1984, une dépression lacustre fut mise en évidence au pied d'une butte témoin située à $10 \mathrm{~km}$ au nord de Shabwa ${ }^{2}$. De nombreuses structures d'habitat et funéraires adossées à cette butte bordaient cette dépression: un des foyers est daté de 7020 ans $\pm 100 \mathrm{BP}^{3}$; matériel de broyage, outillage lithique (grattoirs, armatures bifaciales), obsidienne et fragments d'ouf's d'autruche caractérisent le matériel de surface associé au foyer.

Le Ramlat as-Sab’atayn, ouvert sur le Rub’ al-Khali et frontalier avec l'Arabie saoudite était inaccessible avant la récente réunification des deux Yémen. Afin de définir le cadre

2. INIVAN et ORTI.IFB. $1987: 10$.

3. Gif-9488: tableau 1. 


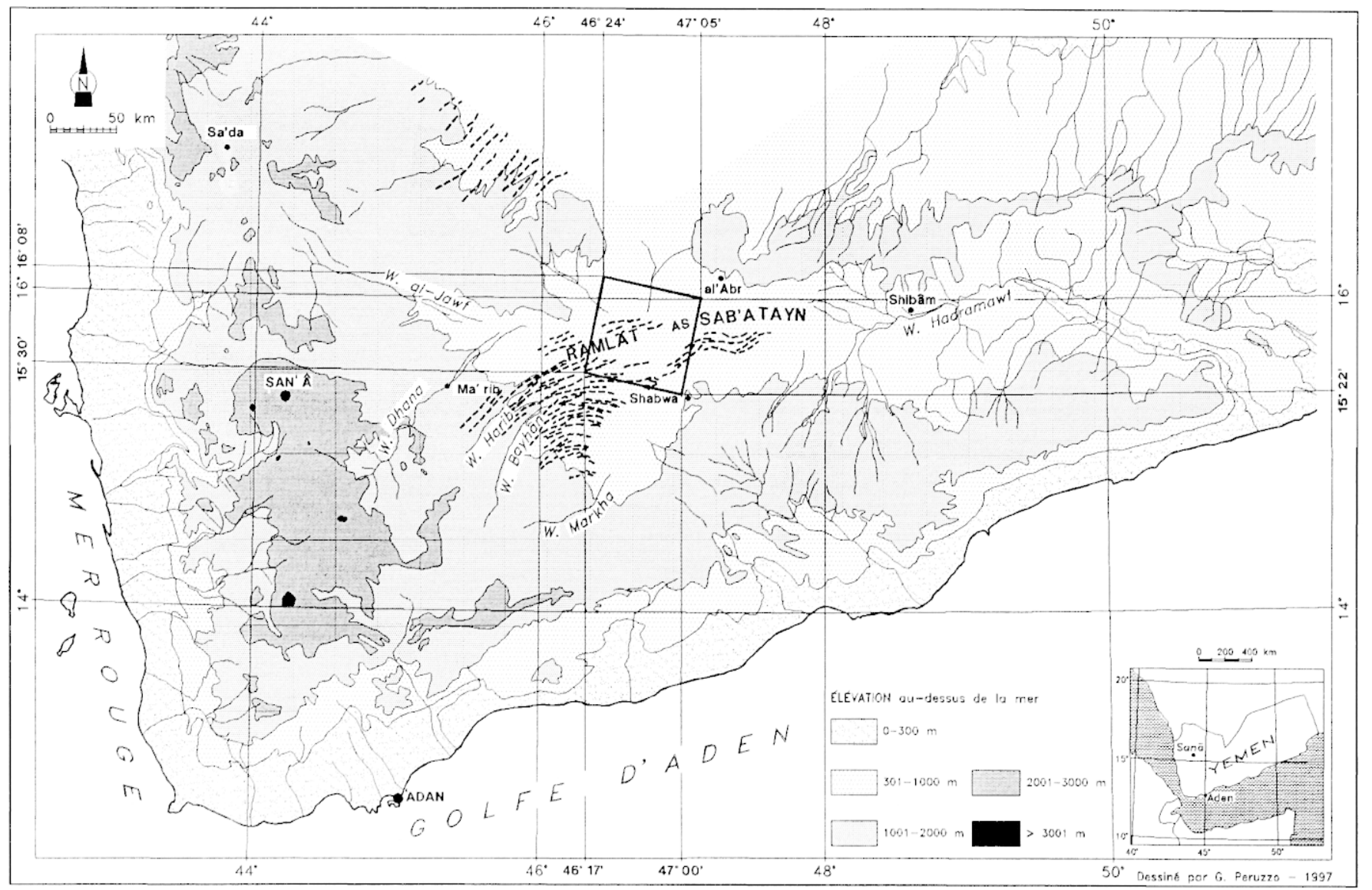

Fig. 1 : Esquisse orographique du Yémen élaboré (B. Marcolongo, CNR/IRPI, Padova, Italie).

naturel de ce bassin et son peuplement et en l'absence de toute cartographic, un programme d'analyses d'images satellitaires et de photographies aériennes fut implanté en collaboration avec B. Marcolongo ${ }^{4}$. L'exploitation des données obtenues à partir des nouveaux satellites Landsat, Soyouz et Spot a permis, surtout à partir de 1990, de prévoir un programme de prospections très précis.

La première cartographie thématique a montré que cette région était autrefois un bassin fluvial où les principaux wâdîs comme les wâdîs Markha, Bayhân, Dhana issus des hautes terres confluaient vers un réseau unique formé des wâdîs principaux, le Jawf et le Hadramawt ${ }^{5}$. Une mission sur le terrain en 1993 permit de contrôler au sol d'une part, le tracé du paléo-cours et d'autre part, l'existence de gisements préhistoriques en relation avec ce potentiel hydrographique. La nature des vestiges identifiés et recueillis, tous en surface,

4. Lacquisition des documents Spot fut réalisée grâce au programme Isis du CNES et financée par le GDR 743 du CNRS. Linterprétation fut effectuée à I'IRPI à Padouc par B. Marcolongo.

5. Cir: ainsi que la conformation de la dépression paléolacustre décelée en 1993 montrant différents états de remplissage, témoignaient en faveur d'une occupation préhistorique dès le Pléistocène confirmant les observations effectuées précédemment sur le pourtour du bassin, surtout dans la région de Shabwa et du Jawf. Les vestiges qui semblent les plus anciens concernent une industrie lithique taillée avec des bifaces de type acheuléen et quelques restes de faune découverts dans la dépression d'al-Hawa en 1993 (fig. $2: 25$ ) : ces restes très fossilisés ont été identifiés par J. Desse ${ }^{6}$; il s'agit pour les gros ruminants d'une faune disparue mais encore présente à l'Holocène au Yémen comme le grand bœuf (Bubalus) ${ }^{7}$. En revanche, la présence d'une dent jugale de cheval est plus surprenante, car l'introduction du cheval en Arabie est admise comme historique et cette dent se trouve hors de l'aire de répartition des chevaux sauvages quaternaires (Equus ferus).

6. Communication personnelle de Jean DEsst. CRA-CNRS. Sophia Antipolis. Valbonne (en cours de publication).

7. GNRCIA et al. 1991: RACHAD, 1994. 
Tableau 1 : Datation ${ }^{14} \mathrm{C}$ sur fover (région de Shabua, al-Hawa et wâdî Markha).

\begin{tabular}{|l|c|c|c|c|}
\hline \multicolumn{1}{|c|}{ Sites } & Age ${ }^{\mathbf{1 4}} \mathbf{C}(\mathbf{B P})$ & Échantillon & Matériel & Numéro laboratoire \\
\hline W. Markha & $8470 \pm 140$ & YE92/4(MKH26) & charbon & Gif-9486 \\
\hline W. Markha & $8470 \pm 145$ & YE92/1(MKH26) & charbon & Gif-9489 \\
\hline Hawa & $7120 \pm 140$ & YE93(ABR1-3) & charbon & Pa- 1520 \\
\hline Quruh Makhabi & $3755 \pm 120$ & Y96.07 & charbonate-hydroxylapatite & Gif-6769 \\
\hline Shabwa & $4800 \pm 400$ & SH84 (16) & charbon & Gif-9488 \\
\hline Shabwa & $5330 \pm 70$ & SH84 (7c) & charbon & Gif-9490 \\
\hline Shabwa & $7020 \pm 100$ & YE92/6(QWD1) & charbon & Gif-9489 \\
\hline Shabwa & $5930 \pm 115$ & YE92/8(IRM2) & YE92/7(IRM2) & \\
\hline Shabwa & $4240 \pm 50$ & & & \\
\hline
\end{tabular}

Une recherche interdisciplinaire fut mise sur pied afin de fournir une chronologie des sites d'occupation et de définir leurs paléoenvironnements en relation avec des séquences sédimentaires. Les informations concernant les changements climatiques dans le sud de la Péninsule arabique sont rares ${ }^{8}$ et les recherches de McClure sur le paléolac de Mundafan ${ }^{9}$, publiées en 1976. restent encore les seules références pour cette région.

Ainsi l'étude des affleurements et des carottages échantillonnés lors de la mission d'octobre 1996 dans la dépression d'al-Hawa apporte la première séquence continentale datée des épisodes hydroclimatiques du Quaternaire récent au sud de la Péninsule arabique. Cette mission a enrichi les données archéologiques en doublant le nombre de sites détectés, qui témoignent d'une longue occupation allant d'un Paléolithique vraisemblablement ancien jusqu'à l'âge du Bronze inclus. pour lequel de nombreuses structures funéraires et d'habitat ont été repérées.

\section{CONTEXTE GÉOGRAPHIQUE ET CLIMATIQUE RÉGIONAL}

Le Ramlat as-Sab'atayn, dont l'altitude moyenne est de 900 mètres. est bordé au sud-ouest par le massif montagneux du socle cristallin et métamorphique des hauts plateaux yéménites qui culminent vers 3000 mètres. A l'Est il est limité par l'immense plateau calcaire du Hadramawt qui s'étend

8. SANLANILIF. 1992.

9. MCCI.l'Rt. 1976. jusqu'au Dhofar. Au Nord, il est séparé du Rub' al-Khali par une série de modestes élévations qui représentent des inselbergs du socle méta-volcanique profondément érodés (fig. 1).

Le climat de cette région est aujourd'hui sub-désertique. marqué par des précipitations n'excédant pas $200 \mathrm{~mm}$ par an et une très intense évaporation ${ }^{10}$. La végétation est de type steppique : sur les dunes. elle est majoritairement herbacée et comprend Panicum turgidum, Leptadenia pyrotechnica, Calligonum comosum et Aristida plumosa associés à Diptengium glaucum et Cyperus conglomeratus de même que des plantes annuelles: Plantago ciliata et Anastatica hierochumtia. Quand ils sont présents. les arbres sont principalement représentés par Acacia tortilis, A. oerfota et A. hamulosa en bordure des escarpements, et par Tamarix nilotica en bordure des wadis. Les sols salés sont caractérisés par la présence de Salsola imbricata et Suaeda aegyptiacall.

\section{CONTEXTE GÉOMORPHOLOGIQUE}

Les travaux géomorphologiques ${ }^{12}$ et les prospections de terrain dans le nord-est du désert du Ramlat as-Sab'atayn située entre $46^{\circ} 15^{\circ}-47^{\circ} 15^{\circ}$ de longitude Est et $\left.15^{\circ} 25^{\circ}-16^{\circ}\right) 5^{\circ}$ de latitude Nord, décrits plus hauts. ont montré que cette région constituait une région clé pour comprendre l'évolution paléoenvironnementale du bassin hydrographique Jawf-Hadramawt à la fin du Quaternaire.

10. WAITtK et al. 1975 .

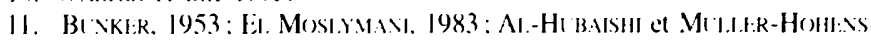
TH:N. 1984.

12. (i.1:1\%101 at al. 1992 


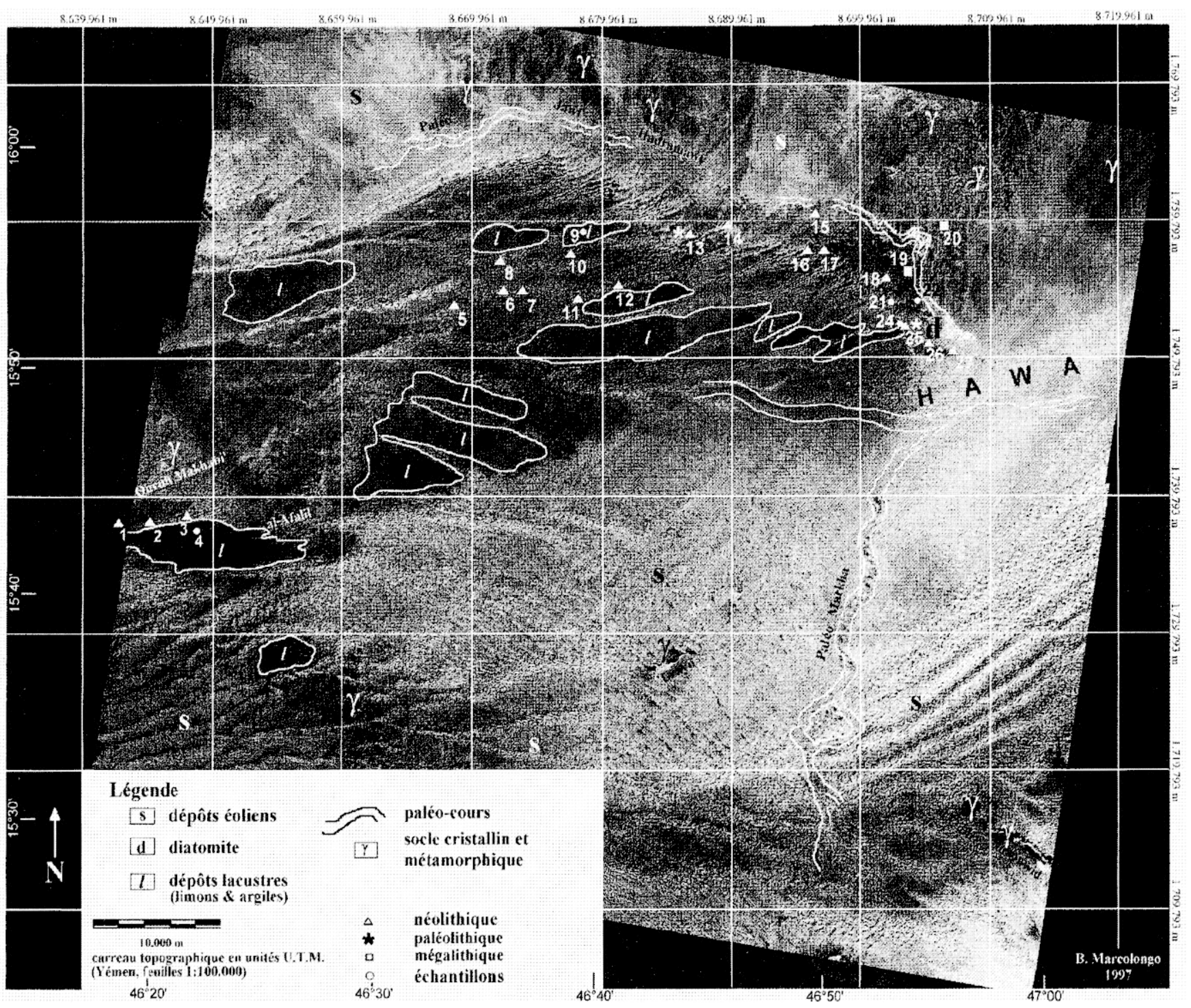

Fig. 2 : Interprétation géomorphologique de la bordure du Ramlat as-Sabatayn fondée sur image SPOT XS rectifiée et géocodée par B. Marcolongo (CNR/IRPI, Padova, Italie).

Les données digitalisées de deux images SPOT XS élaborées à partir d'un logiciel (GEOimage) ont permis la réalisation de cartes au $1: 25000$ de la zone à prospecter et l'interprétation des dépressions interdunaires (fig. 2). La reconnaissance de l'étendue des dépôts lacustres en relation avec le système paléofluviatile du Jawf-Hadramawt, jusque là inconnue, a déterminé la prospection géoarchéologique d'octobre 1996.

Trois grands ensembles de dépressions identifiés sur image ont été contrôlés (fig. 2), le premier près de l'inselberg de Quruh Makhabi', le second près de l'inselberg d'al-Afalil et le troisième vers la grande dépression d'al-Hawa. Dans la zone d'al-Hawa, des dépôts de morphologie lacustre avaient été reconnus dès 1993. Ils sont localement plissés et/ou basculés, probablement en relation avec un épisode néotectonique ou une remontée de diapires reliée aux complexes diapiriques présents sur les bordures ouest et sud-est du Ramlat as-Sab'atayn.

Un paléocours d'eau, individualisé sur les images SPOT par sa réflectivité spécifique, a été reconnu sur le terrain par la présence d'étroites bandes de graviers et de cailloux (lithotypes du bassin hydrographique du Jawf) et d'alignements de végétation, buissons et arbustes. Des traces d'autres paléocours, identifiés sur les images, sont indiquées sur la 
figure 2. On remarque que le paléo Markha converge au sud vers la dépression d'al-Hawa, recoupant clairement dans sa dernière partie les dunes longitudinales, suggérant une activité fluviatile récente.

Les analyses granulométriques ont été faites sur 4 échantillons (fig. $2: 4,9,21,24)^{13}$; les échantillons 21 et 24 sont des silts avec une quasi absence de sable et d'argile; les échantillons 4 ct 9 sont aussi des silts avec une composante considérable de sable fin. Il semble donc que la bordure nord du paléo-lac ait été plus profonde. avec une franche sédimentation lacustre, que la partie sud caractérisée par des sédiments terrigènes. Cette différence pourrait être en relation avec la branche majeure du paléocours Jawf-Hadramawt. La localisation et la distribution des sites semblent en étroite relation avec la bordure nord-est du paléolac.

\section{OCCUPATIONS PRÉHISTORIQUES}

\section{LE PALÉOLITHIQUE}

Concernant le Paléolithique au Yémen, il est encore difficile d'estimer son origine et son développement. Rappelons que seul le site d'al-Guzah au bord d'un affluent de rive droite du wâdî Hadramawt, a été daté de $700(0)()$ ans et qu'il possède une industrie de type archaïque à sa base ${ }^{14}$ ce qui pose alors le problème suivant: Homo erectus avait-il déjà colonisé le sud de l'Arabie? Trente-sept concentrations d'industries lithiques taillées à caractères archaïques, de type oldowayen (polyèdres, pics, galets taillés, burins) ont été relevées en surface dans la région d'Aden; elles témoigneraient, selon les auteurs, d'un passage de l'Afrique très précoce par le Bab el-Mandeb ${ }^{15}$.

Bien que toujours non datée, l'occupation paléolithique trouvée en surface, est bien documentée dans le Ramlat as-Sab'atayn (région de Shabwa, du Jawf, dans la dépression d'al-Hawa). C'est soit une industrie à caractère acheuléen avec bifaces. racloirs, denticulés, très souvent taillée sur quartzite qui est présente dans la dépression d’al-Hawa, soit une industrie à bifaces associée à un débitage Levallois (éclats. pointes et lames) comme dans la région de Shabwa et du Jawf.

13. Alberto PAI.MIr:RI, CNR/STABC. Rome : en cours de publication. 14. A.MIKHANON, 1994.

15. WHALEN and SCHATTL. 1997.
Les caractères techniques et typologiques des industries lithiques du Paléolithique supérieur habituellement observés dans l'Ancien monde ne semblent pas exister dans toute l'Arabie. En l'absence de tout marqueur chronologique, il est hors de question de préciser la fin du Paléolithique en se fondant uniquement sur des caractères techniques de la pierre taillée.

\section{LE NÉOLITHIQUE}

Concernant la néolithisation. il est aussi très difficile de préciser l'apparition de ce phénomène au Yémen. Néanmoins, le concept de néolithisation étant suffisamment élaboré et complexe $^{16}$, on peut y faire référence au Yémen malgré la rareté des documents essentiels concernant la domestication des animaux et des plantes ainsi que l'apparition tardive de la technique céramique.

Les sondages et les fouilles dans des couches néolithiques ont commencé depuis quelques années, essentiellement dans les montagnes, près de Sa'na ${ }^{17}$, à l'est de Sa'na dans le Khawlan, près de Dhamar ${ }^{18}$ au sud. près de Sa' da ${ }^{19}$ et dans la plaine de la Tihamâ ${ }^{2()}$ apportant les premières données concernant la domestication des animaux. Pour le Ramlat as-Sab atayn, dès les environs de $7000 \mathrm{BP}^{2}$. on peut élargir la notion de "néolithique» à l'apparition de phénomènes techno-économiques nouveaux comme le broyage (fig. 3), la circulation de matières premières telle l'obsidienne ou de coquillages marins (cauri, Engina mendicaria: fig. $4: 11,12$ ). On observe de plus, une diversification dans les armes de jet avec une variété typologique (forme et dimension) et technique traduisant une évolution dans l’acquisition du gibier. La légèreté, les ailerons des toutes petites pointes de flèche suggèrent l'utilisation probable de l'arc. Les techniques de taille sont caractérisées par l'utilisation fréquente du percuteur dur pour la taille des supports, le plus souvent des éclats, alors que la retouche par pression à enlèvements obliques très parallèles détermine le façonnage final des pointes de flèche avec ou sans aileron: cette observation vaut aussi pour les industries «néolithiques» du Rub" al-Khali22 (fig. 4).

Cependant les appellations comme "Arabian Bifacial Tradition" sont dangereuses : le caractère bifacial qui s’applique en général à des armes de jet. implique tout au plus la

16. Alri:NCHe et CalTix, 1989.

17. KNIINIIT. 1996.

18. FH:Dt:I.1. 1984.

19. WII.KINSON. EIIHN and GIBSON. 1997.

20. Tosi. 1986.

21. Tableau 1: (jif-9488.

22. EDENS. 1988. 

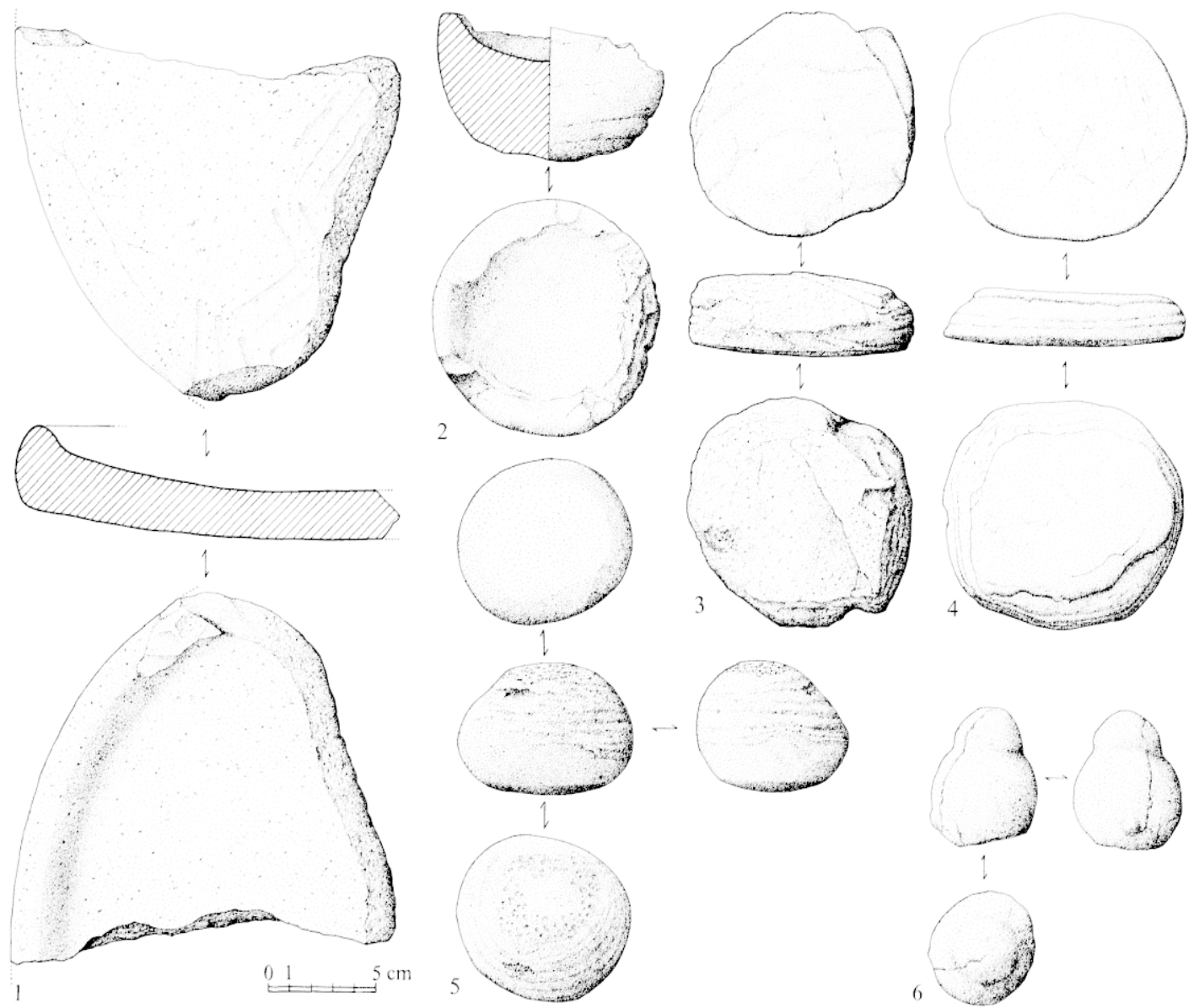

Fig. 3 : Matériel de brovage et ustensile de pierre: I, meule arec rebord: 2, bol en pierre;

3, 4, petites méules plates en grès: 5, molette piquetée: 6, molette? (dessin G. Monthel).

présence de chasseurs mais ne peut être pris en compte comme marqueur chronologique et encore moins définir des cultures en omettant tout le reste de l'équipement. Il est encore prématuré de préciser le moment de ces apparitions et de décider s'il s'agit d'un emprunt ou d'une évolution sur place devant la rareté des datations et d'un matériel stratifié. Quant au débitage laminaire tel qu'il est pratiqué au ProcheOrient et tel qu'il a été identifié sur les rives du Golfe (type naviforme associé à des armatures caractéristiques façonnées sur lame) ${ }^{23}$, il n'a jamais été reconnu au Yémen.

Du point de vue chronologique deux foyers construits ont été relevés dans une coupe naturelle située dans un affluent du wâdî Markha, ils ont fourni les dates les plus anciennes que nous connaissions, soit $8470 \pm 140 \mathrm{BP}^{24}$, l'industrie lithique recueillie au pied de la coupe, est composée de quartz taillé sur éclat sans outil et sans technique caractéristiques.

23. INI7AN, $1988: 33-54$.

24. Tableau 1: (Gif-9486 et Gif-9483.

\section{L'ENVIRONNEMENT RÉGIONAL DE L'HOMME À L'HOLOCÈNE : L'ENREGISTREMENT LACUSTRE D'AL-HAWA}

\section{LA SÉQUENCE SÉDIMENTAIRE}

Les sédiments holocènes ont ćté prélevés dans deux secteurs différents de la dépression: Hawa 2 et 4 . Ils sont formés de deux ensemble: L'unité $B$ à la base de la série Hawa 4 est composée d'un silt calcaire non consolidé, noir, sur $1 \mathrm{~m}$ d'épaisseur. De fins niveaux enrichis en sable ou en argile s'observent localement. L'unité $A$ au sommet de la série Hawa 4 (sur $20 \mathrm{~cm}$ d'épaisseur) ainsi que dans la série Hawa 2 (sur $80 \mathrm{~cm}$ d'épaisseur) est composée de sédiments compacts blancs à gris formés d'une alternance de silts calcaires à diatomées et à coquilles (fig. $2: 21,22$ ). 

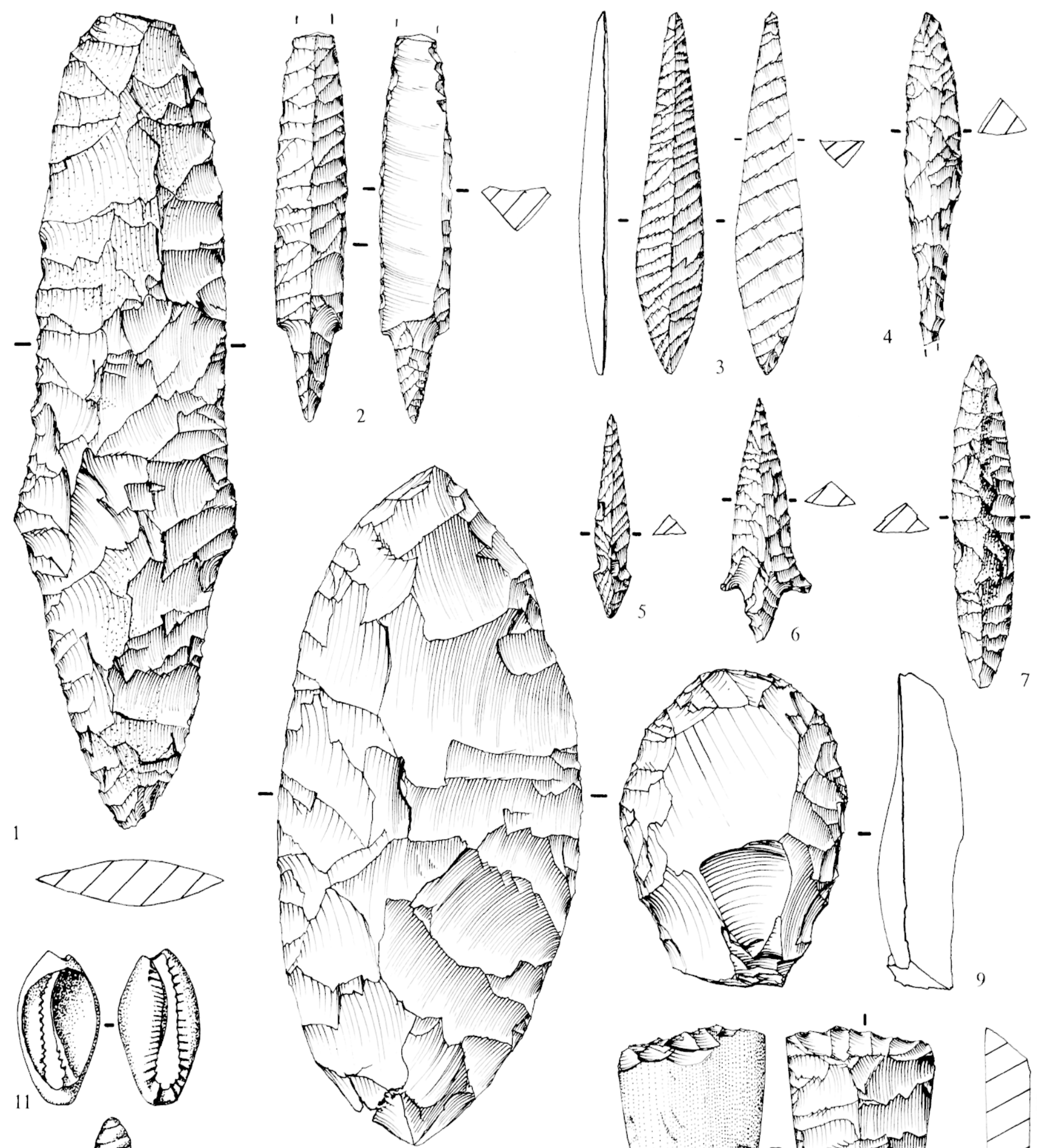

12

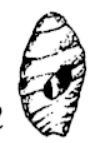

13

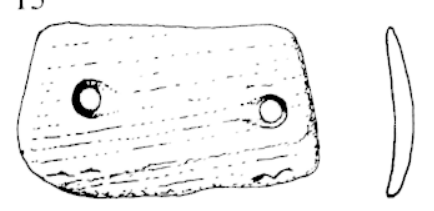

$s$

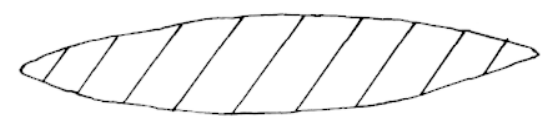

$\begin{array}{llllll}0 & 1 & 2 & 3 & 4 & 5 \mathrm{~cm}\end{array}$
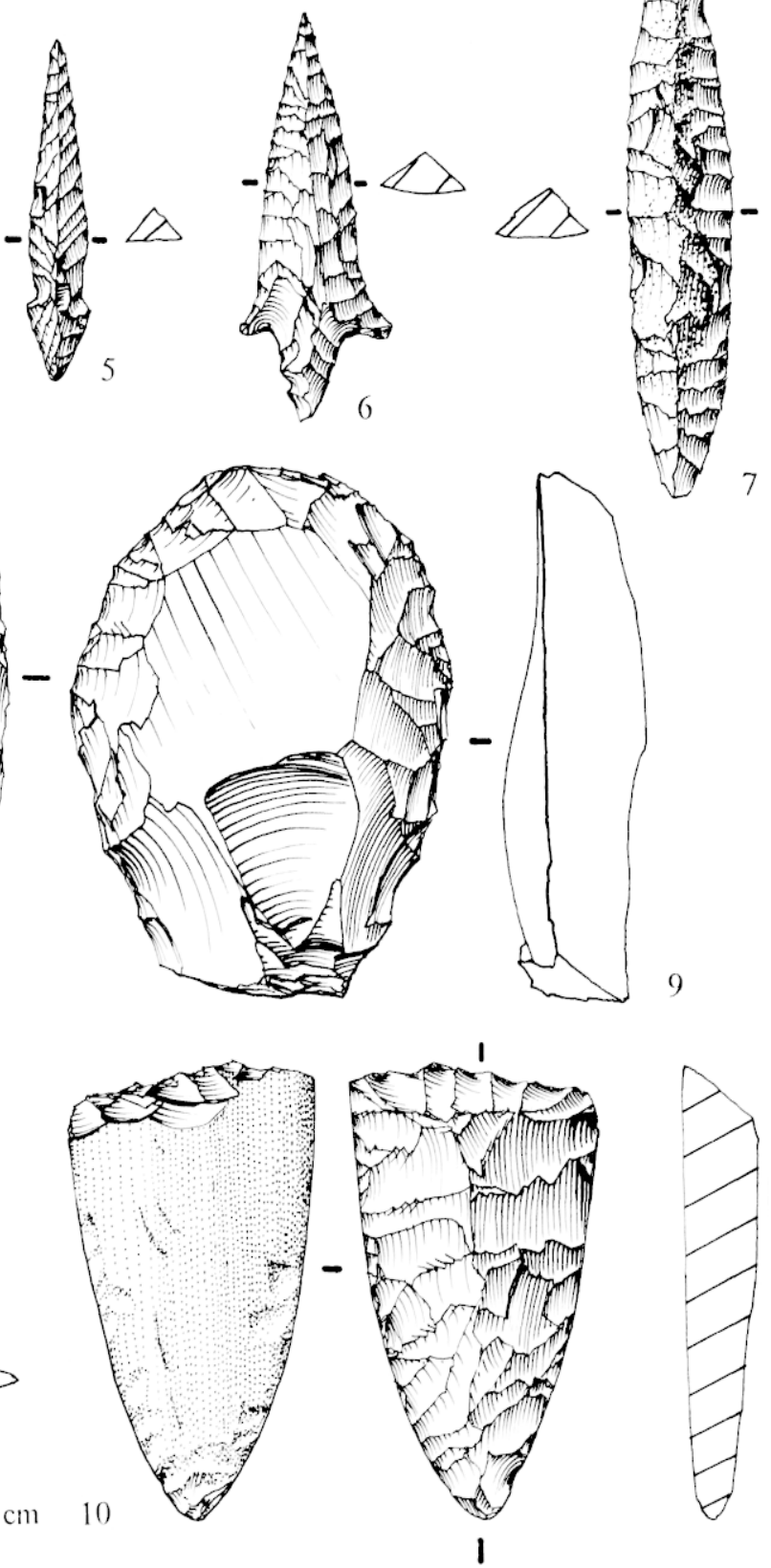

Fig. 4 : Industrie lithique des sites de la région d'al-Hawa, site 17: 1. piéce bifaciale façonnée au percuteur tendre (type "poignard "): 2. 3. 4. 5, 6. 7. armatures bifaciales, retonchées par pression en écharpe 1.3 et 5): 8. piéce foliacée bifaciale en quart-ite taillée par percussion: 9. grattoir sur éclat en silex: 10, petite hache taillée en silex puis polie sur une face: 11, rauri: 12. Engina mendicarial perforcé : 13, petite plaque de narre perforée (dessin $M$. Redurom). 


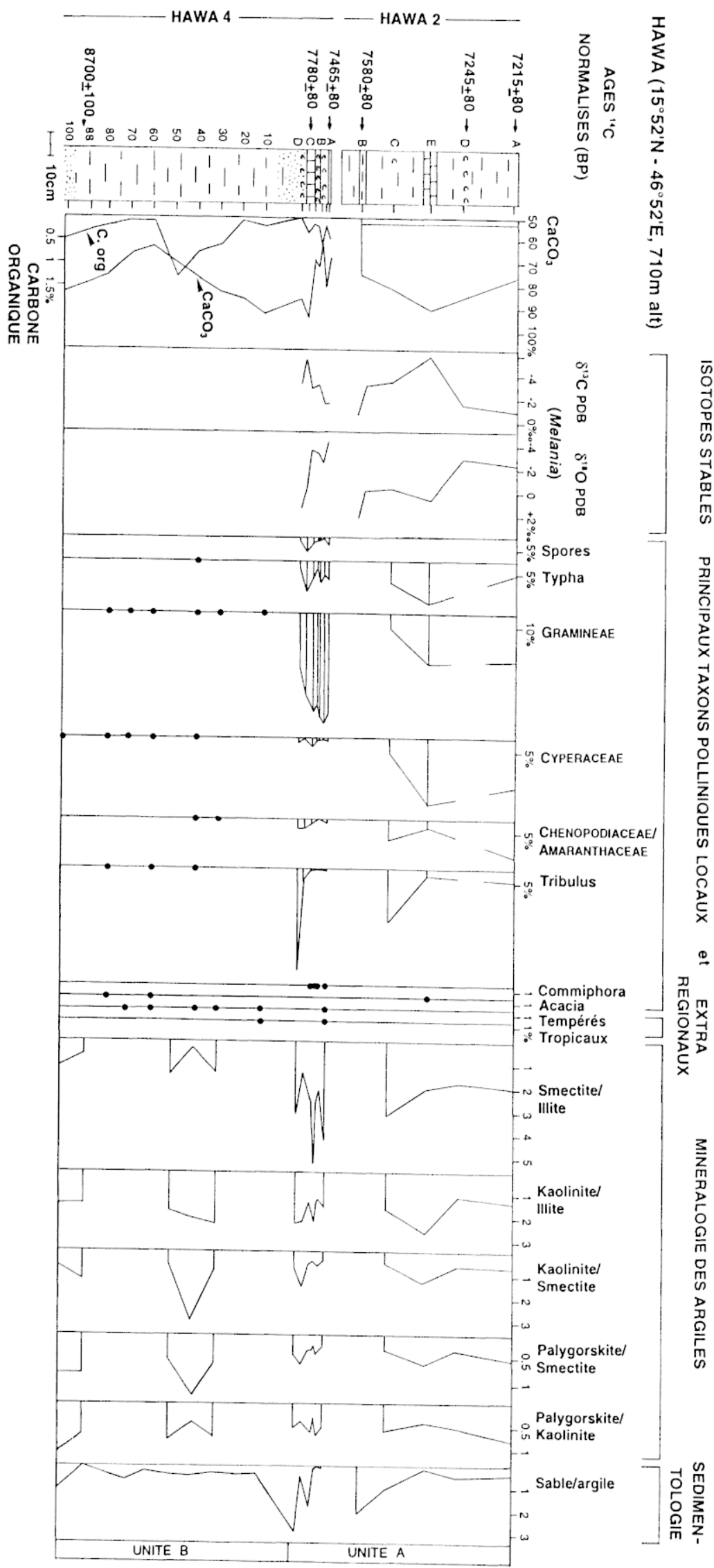

Fig. 5 : Sỵthèse des données sédimentologiques, minéralogiques, isotopiques et palynologiques du site d'al-Hawa. Les séquences Hawa 2 et 4 sont placées une au dessus de l'autre en fonction de leur âge. Les âges ${ }^{14} \mathrm{C}$ suggèrent leur léger recouvrement. La série sédimentaire complète de remontée des nappes et/ou de l'alimentation de la dépression (unité B) et lacustre (unité $A$ ) représente environ deux mètres d'épaisseur. 
Tableau 2: Datation ${ }^{i 4} \mathrm{C}$ des séries Hana 2 et 4.

\begin{tabular}{|c|c|c|c|}
\hline $\operatorname{Age}{ }^{14} \mathrm{C}(\mathrm{BP})$ & Échantilon & Matériel & Numéro de laboratoire \\
\hline $7245 \pm 80$ & Hawa 2D & Melania & $\mathrm{Pa}-1531$ \\
\hline $7580 \pm 80$ & Hawa 2B & $\ll$ & $\mathrm{Pa}-1532$ \\
\hline $7215 \pm 80$ & Hawa $2 \mathrm{~A}$ & « & $\mathrm{Pa}-1533$ \\
\hline $7465 \pm 80$ & Hawa $4 \mathrm{~A}$ & « & $\mathrm{Pa}-1559$ \\
\hline $7810 \pm 200$ & Hawa $4 \mathrm{C}$ & « & $\mathrm{Pa}-1560$ \\
\hline $7780 \pm 80$ & Hawa $4 \mathrm{C}$ & " & $\mathrm{Pa}-1567$ \\
\hline $8700 \pm 100$ & Hawa $4 \mathrm{~T}(-88 \mathrm{~cm})$ & M. organique & Pa- 1582 \\
\hline
\end{tabular}

\section{LE CONTEXTE CHRONOLOGIQUE}

Les mesures de radiocarbone ont été effectuées sur des coquilles de Melania et soigneusement nettoyées aux ultrasons afin d'éliminer toute trace de sédiment détritique. Elles donnent des âges normalisés ${ }^{25}$ qui se situent entre 7780 et 7215 BP (Tableau 3). Nous présumons que la précipitation du carbonate de ces coquilles s'est effectuéc à l'équilibre ou proche de l'équilibre avec le $\mathrm{CO}_{2}$ atmosphérique, soit directement soit par l'intermédiaire de la biomasse végétale. Dans la vaste dépression d'al-Hawa. les temps de résidence des eaux dans les systèmes lacustres ont dû être suffisamment longs pour admettre que les âges ${ }^{1+} \mathrm{C}$ calculés sont légitimes.

La mesure du radiocarbone contenu dans la matiere organique algaire à la base de l'unité $\mathrm{B}$ a donné un âge normalisé de $87(0) \pm 100$ BP (Tableau 2).

\section{L'ENVIRONNEMENT RÉGIONAL: IMPLICATIONS HYDROLOGIQUES ET CLIMATIQUES}

Les assemblages polliniques permettent de reconstituer I'histoire locale et régionale de la dépression d'al-Hawa lors du développement lacustre. Les grains de pollens sont très rares ou absents de l'unité $B$ et de certains niveaux d'Hawa 2 dans l'unité A; ces derniers étant fortement dégradés en affleurement. Dans l'unité A. les comptages ont porté sur 162 à 1891 grains selon les niveaux et 43 taxons ont pu être déterminés (Tableau 3). Ces taxons appartiennent en majorité à la flore locale et régionale actuelle du site. On note cepen-

25. STITLR and P()A(H. 1977. Par convention internationale. les ages ${ }^{1+} \mathrm{C}^{\circ}$ doivent étre calculés en utilisant la période de demi-vie consentionnelle de 5.568 ans et une normalisation des d d $^{1:} \mathrm{C}$ égale $\pm 25.0 \%$ basé sur la relation de CralGi. 1954. dant la présence de taxons "exotiques» en provenance soit d'écosystèmes tropicaux (3 taxons) ou tempérés (5 taxons) qui donnent l'indication d'un transport de longue distance des grains de pollen.

\section{L'unité B}

La base de la séquence Hawa 4 est caractérisée du point de vue sédimentologique par l'abondance des grains anguleux carbonatés qui représentent entre 60 et $90 \%$ du sédiment total. La quasi-absence de minéraux argileux dans la fraction fine résulte d'une faible altération de surface. conséquence probable d'un environnement encore aride et d'une érosion intense par les vents. Lorsque les minéraux argileux sont présents en quantité suffisante pour être analysés. ils contiennent entre autre de la palygorskite, minéral abondant dans les affleurements d'Arabie centrale où il est parfois associé à la smectite. et transporté par les vents jusqu'en mer d ${ }^{\circ}$ Arabie $^{26}$. Chlorite et illite sont associés aux interstratifiés et à la kaolinite: la présence de ce minéral qui provient de zones bien drainées et dont on retrouve la trace dans les apports fluviatiles holocènes du Yémen ${ }^{27}$ suggère une origine proximale et le développement d'altérations dans le bassin-versant d'Hawa.

Lorsqu ils sont présents, les grains de pollen correspondent à une association steppique proche de l'actuelle. Toutefois. la présence notable de grains exotiques, en provenance d'écosystèmes tempérés (Betula, Carpinus...) et d’altitude (Cedrus) actuellement étendus au Nord de la Péninsule arabique (Moyen-Orient. Zagros) ne peut sexpliquer par une modification paléogéographique sur le site même d’al-Hawa. Ces grains allochtones sont le reflet d'un transport éolien accru

26. SIRO(KO) and LAN(it: 1991.

27. SiRocko et al. 1993. 
Tableau 3 : Liste des taxons polliniques représentés dans les sédiments des séquences Hawa 4 et 2; * = type pollinique regroupant plusieurs espèces, genres ou familles.

\begin{tabular}{|c|c|c|}
\hline \multicolumn{2}{|c|}{ Plantes locales } & \multirow{2}{*}{$\begin{array}{c}\text { Plantes extra-régionales } \\
\text { (Arboreal Pollen) }\end{array}$} \\
\hline (Arboreal Pollen) & (Non-Arboreal Pollen) & \\
\hline $\begin{array}{l}\text { Acacia } \\
\text { COMBRETACEAE } \\
\text { Commiphora } \\
\text { Maerua* } \\
\text { RHAMNACEAE }\end{array}$ & $\begin{array}{l}-1 \text { - Plantes herbacées } \\
\text { Aerva javanica } \\
\text { Aerva lanata } \\
\text { Artemisia } \\
\text { Cassia } \\
\text { CARYOPYLLACEAE } \\
\text { Centaurea perrottetii* } \\
\text { CHENOPODIACEAE } \\
\text { COMPOSITAE tubul. } \\
\text { COMPOSITAE ligul. } \\
\text { CONVOLVULACEAE } \\
\text { CRUCIFERAE } \\
\text { CYPERACEAE } \\
\text { Dipterygium } \\
\text { Ephedra } \\
\text { Fagonia } \\
\text { GRAMINEAE } \\
\text { LABIATAE } \\
\text { Plantago } \\
\text { Polygala } \\
\text { RESEDACEAE } \\
\text { Rumex } \\
\text { Tribulus } \\
\text { URTICACEAE* } \\
\text { Typha } \\
\text { Zygophyllum } \\
\text { - 2 - Fougères et Mousses } \\
\text { Azola } \\
\text { Ceratopteris } \\
\text { Pteris } \\
\text { Spore monolète } \\
\text { Spore trilète } \\
\text { Anthocerothaceae }\end{array}$ & $\begin{array}{l}-1-\text { Tempérées } \\
\text { Betula } \\
\text { Carpinus } \\
\text { Cedrus } \\
\text { Fagus } \\
\text { Quercus } \\
-2-\text { Tropicales } \\
\text { CAESALPINIACEAE } \\
\text { Dodonaea } \\
\text { Juniperus }\end{array}$ \\
\hline
\end{tabular}

lié à une circulation d'alizé intense. Des grains d'origine tropicale (Caesalpiniaceae) ont également été dénombrés. Ils ne peuvent qu'avoir une origine méridionale. Leur présence à al-Hawa serait alors liée aux précipitations décrites plus haut.

\section{L'unité A}

L'unité A enregistre l'installation d'un lac d'eau douce qui subit plusieurs variations de niveau. L'instabilité hydrodynamique est attestée par les fluctuations de la distribution granulométrique du sédiment qui montre en alternance des accroissements importants des fractions sableuses et argileuses. Les sédiments lacustres étant tronqués par déflation postérieure, il est probable que le lac a duré au delà de 7200 BP.
Les caractères sédimentologiques et minéralogiques montrent l'importance croissante de l'humidité et du ruissellement dans l'ensemble du bassin versant d'al-Hawa. L'importance décroissante des carbonates détritiques résulte probablement d'un effet de dilution, lié au développement de l'activité biologique dans le lac (indiqué par l'abondance des diatomées) ainsi qu'à celui de l'altération et des néogenèses (minéraux argileux) dans le bassin versant. Le principal trait de l'association minéralogique est l'accroissement de la teneur en smectite témoignant du développement de formations pédogéniques etou de néogenèses dans les zones aval du bassin versant, plaines d'inondation bordant le milieu lacustre. La kaolinite, plus abondante que précédemment, suggère le développement d'altérations sur les reliefs et la persistance des apports depuis les zones amont. Enfin, la persistance de l'illite et des interstratifiés, en association avec les apports détritiques 
carbonatés indique la poursuite d’apports issus de secteurs peu ou pas altérés.

Les conditions générales du milieu. steppiques, sont confirmées par les résultats palynologiques qui montrent la prédominance des taxons herbacés: Gramineae et Tribulus, Cyperaceae, Artemisia; les grains de pollen d'arbres (Acacia. Commiphora) n'apparaissent que ponctuellement en pourcentages toujours inférieurs à 1 . Cette association témoigne d'un environnement semblable à l'actuel mais probablement plus dense qu'aujourd'hui en raison de la présence des arbres sensiblement mieux représentés dans les spectres fossiles que dans les actuels du Ramlat as-Sab'atayn ${ }^{28}$ ou du Rub' alKhali $^{29}$.

La présence de Typha et des spores de fougères en pourcentages notables indique en outre la proximité d'un plan d'eau douce permanent. La variation des pourcentages des principaux taxons suggère l'évolution suivante : la base de la série Hawa 4 où les pourcentages de Tribulus sont importants reflète des conditions générales arides encore très marquées au tout début de la séquence lacustre; les fortes conditions hydrodynamiques sont indiquées par la proportion importante de la fraction sableuse. Ensuite, l'apparition des spores de fougères et des grains de Typha ainsi que le développement de la smectite démontrent l'humidification du site, probablement saisonnière, qui perdure tout au long de la séquence recoupée par les séries Hawa 4 et 2. Tribulus, Chenopodiaceae-Amaranthaceae et Cyperaceae prennent une importance accrue dans la séric Hawa 2 tandis que les spores de fougères disparaissent et les pourcentages de Gramineae diminuent. $\mathrm{Ceci}$, de même que la plus faible proportion de smectite et l'accroissement de la palygorskite, suggère une évolution vers des conditions sensiblement plus arides que précédemment.

Durant cette phase lacustre, des oscillations paléohydrologiques sont enregistrées par les variations des pourcentages de Typha et des mesures ${ }^{18} \mathrm{O}$ et ${ }^{13} \mathrm{C}$ sur les carbonates. Typha appartient à une population hydrophile qui se développe lorsque le niveau du lac est bas, favorisant l'extension d'importantes zones humides en bordure. Ses valeurs maximales (dans les échantillons $\mathrm{E}$ d'Hawa 2 et $\mathrm{Cb}$ d'Hawa 4) correspondent à la décroissance des valeurs de $\delta^{13} \mathrm{C}$ qui reflète les apports de $\mathrm{CO}_{2}$ produit par la respiration et la décomposition de la matière organique. L'accroissement corrélatif des valeurs de $\delta^{18} \mathrm{O}$ répond, quant à lui, à une évaporation intense des eaux du lac. Le rapport entre ces deux valeurs ${ }^{18} \mathrm{O}$ et ${ }^{13} \mathrm{C}$, s'inverse lorsque le niveau du lac est haut.

\section{INTERPRÉTATION}

Nous interprétons les données recueillies sur le site d'alHawa comme l'enregistrement continental du maximum humide lié au renforcement de la mousson indienne d'été à 1Holocène ancien. La base de la séquence correspond à la mise en eau de la dépression dans un contexte aride encore très marqué, dès $8700 \mathrm{BP}$. La mise en eau de la dépression aboutit à l'installation d'un véritable lac d'eau douce qui dure au moins 50() ans. Le lac d'al-Hawa est tardif par rapport à l'augmentation progressive de l'humidité enregistrée dès 14300 BP dans les sédiments océaniques prélevés au large de l'Arabie ${ }^{30}$; le maximum de la mousson indienne étant atteint entre 8850 et 7850 BP. Le temps de réponse du système hydrologique d'al-Hawa à cette évolution est sans doute lié à l'aridité du milieu. Celle-ci étant liée à l'intensité de la circulation d'alizé démontrée par la palynologie et la sédimentologie qui enregistre des apports significatifs depuis les écosystèmes tempérés. L'humidité induite par la mousson indienne n'a, par ailleurs, jamais été suffisante à al-Hawa pour modifier profondément l'écosystème qui garde une affinité sub-désertique tout au long de la période lacustre avec probablement une couverture ligneuse plus dense que l'actuclle.

\section{CONCLUSION}

Les séquences holocènes d’al-Hawa appartiennent à la phase lacustre reconnuc également dans le Rub'al-Khali à l'Holocène ancien (entre 8800 ) et $6100 \mathrm{BP})^{31}$ et qui témoigne de la sensibilité du sud de la péninsule arabique aux variations de la mousson indienne. Des populations préhistoriques se sont installées sur les rives du lac, elles sont repérées par les foyers encore visibles et un matériel lithique qui s'apparente aux industries du Rub'al-Khali (armatures de jet et matériel de broyage) ainsi que la présence de la retouche par pression qui confirme l'unité technique du matériel. Toutefois il reste à préciser l'apparition de la retouche par pression et sa relation avec le Proche-Orient. A ce matériel lithique s'ajoutent des fragments d'reuf d'autruche, des coquillages marins, des os d'animaux etc. 
D'autres phases lacustres (en cours d'analyses) ont été identifiées qui pourraient correspondre aux épisodes hydroclimatiques plus anciens du dernier interglaciaire et correspondre à l'occupation paléolithique dont les industries n'ont malheureusement pas été repérées en stratigraphie.

Les premiers résultats de ces travaux menés dans la région lacustre d'al-Hawa dans le désert du Ramlat as-Sabat'ayn et le long du paléocours Jawf-Hadramawt démontrent la nécessité et l'efficacité d'une recherche interdisciplinaire sur le terrain pour comprendre l'origine et l'évolution du peuplement du sud de l'Arabie.

\section{REMERCIEMENTS}

Nous tenons à remercier les autorités compétentes du Yémen, $\mathrm{M}$. Yussuf Abdallah, Président de l'Organisme général des Antiquités, des Musées et des Manuscrits de la République du Yémen, M. Ahmad Shamsan, Directeur de l'Archéologie qui ont autorisé et facilité nos travaux dans une région difficile d'accès. A Sana'a, nous avons bénéficié de l'aide précieuse et de l'accueil chaleureux du Centre français d'études yéménites et tout particulièrement de son directeur Franck Mermier.

A.-M. L. remercie J.-P. Cazet (CNRS-Paris) pour l'assistance technique.

Les missions de terrain ont été financées par le ministère des Affaires étrangères.

Marie-Louise INIZAN

ERA 28 (UPR 7549), MAE (Maison R. Ginouvès) 21, rue de l'Université, 92023 Nanterre cedex inizan@mae.u-paris10.fr

Anne-Marie LEZINE

URA 1761-CNRS, "Paléontologie et Stratigraphie" Université Paris VI, Jussieu boîte 106 75252 Paris cedex 05 lezine@ccr.jussieu.fr

Bruno MARCOLONGO

IRPI, CNR, Corso Stati Uniti 4 35020 Camin - Padova Italie bruno@irpil.geo.pd.cnr.it

Jean-François SALIEGE

LODYC, Université Paris VI, Jussieu, boûte 100 75252 Paris cedex 05 ifs@lodyc.jussieu.fr

Christian ROBERT Frédéric WERTH

Centre d'Océanographie de Marseille Université Aix-Marseille II, Luminy case 901 13288 Marseille cedex 9 Christian.Robert@com.univ-mrs.fr

\section{BIBLIOGRAPHIE}

Al-Hlbaishi A. and Muller-Hohenstein K.

1984 An introduction to the vegetation of Yemen. Eschborn: GTZ.

AMiKHANOV KH.

1994 Research on the Palaeolithic and Neolithic of Hadhramaut and Mahra. Arabian Archaeology and Epigraphy 5 : 217-228.

AURENCHE O. and CAUVIN J. (eds.)

1989 Néolithisations. BAR Int. Ser. 516: Oxford.

BRETON J.-F.

1992 Rapports préliminaires (Fouilles de Shabwa. II). I.F.A.P.O., publ. hors série $\mathrm{n}^{\circ} 19$ Paris : Geuthner.

BUNKER D.G.

1953 The South-West borderlands of the Rub' Al Khali. The Geographical Journal 119, 4 : 420-430.

Cleuziou S., Inizan M.-L. et Marcolongo B.

1992 Le peuplement pré- et protohistorique du système fluviatile fossile du Jawf-Hadramawt au Yémen (d'après l'interprétation d'images satellite, de photographies aériennes et de prospections). Paléorient 18,2: 5-29.

Cralg H.

1954 Carbon 13 in plants and the relationships between carbon 13 and carbon 14 variations in nature. J. Geol., $62: 115-149$.

EDENS C.

1988 The Rub al-Khali 'Neolithic' Revisited: the View from Nadgan. In : Potts D.T. (ed.) : Araby the blest : 15-44: Copenhagen: The Carsten Nieburhr Inst. Of Ancient Near Eastern Studies, University of Copenhagen.

El Moslymani A.P.

1983 History of climate and vegetation in the Eastern Mediterranean and the Middle East from the Pleniglacial to the mid-Holocene. University of Washington: Ph-D. Thesis.

FEDELE F.G.

1990 Man, land and climate : Emerging interaction from the Holocene of the Yemen Highlands. In : BotTeMA S., ENTJEs-NiEBORGi G.T. and Van ZEIST W. (eds) : Man's Role in the Shaping of the Mediterranean Landscape : 31-42. Rotterdam : Balkema.

Garcia M., Rachad M., Hadjouis D., Inizan M.-L. et Fontugne M.

1991 Découvertes préhistoriques au Yémen. Le contexte archéologique de l'art rupestre de la région de Saada. Comptes Rendus de l'Académie des Sciences de Paris. 313 série II : 1201-1206.

INIZAN M.-L. (ed.)

1988 Préhistoire à Qatar. Paris: ERC.

INIZAN M.-L. et ORTLIEB L.

1987 Préhistoire dans la région de Shabwa au Yémen du sud (R.D.P. Yemen). Paléorient 13,1: 5-21.

KALLweIT H.

1996 Neolithische und Bronzezeitliche Besiedlung im Wadi Dhahr, Republik Jemen. Eine Untersuchung auf der Basis von Geländebegehungen und Sondagen. Freiburg: Albert-Ludwigs-Universität, Inaugural Dissertation.

MarColongo B

1996 Modelli di utilizzo della risorse idriche nello Yemen interno dall 'eta' del bronzo al periodo sudarabico. Proceedings of the first international conference on the conservation and exploitation of the archaeological heritage of the Arabian Peninsula, Arabia Antiqua, Roma, 28-30 mai 1991, Serie orientale Roma LXX, I : 179-187. 
MCCLt'RE. H.A.

1976 Radiocarbon chronology of late Quaternary lakes in the Arabian desert. Nature 263: 755-756.

RACHAD M.

1994 L'art rupestre et son contexte préhistorique au Yémen dans la région de Sâdaa et Radâ (R.Y.). Université de Paris-I-Sorbonne: Thèse de Doctorat.

SIROCKO F. and LANGE H.

1991 Clay-mineral accumulation rates in the Arabian Sea during the late Quaternary. Marine Geology 97 : 105-119.

Sirocko F., Sar.vthein M., Erle.nkeuser H., Lange H., Ar.nold M. and DiPLESSY J.C.

1993 Century-scale events in monsonal climate over the past 24,000) years. Nature $364:$ 322-324.

Stuiver M. and POLACh H.A.

1977 Discussion reporting of ${ }^{14} \mathrm{C}$ data. Radiocarbon 19, 3:355-363.
Tosı M.

1986 Archaeological activities in the Yemen Arab Republic. 1986. Survey and excavations on the coastal plain (Tihamah). East and West 36, $4: 4(0)-414$.

Watter H.. Har.ickell. E. and Mi El.ler-Dombois D.

1975 Climate-diagram maps of the individual continents and the ecological climatic regions of the Earth. Supplement to the vegetation monographs. Berlin : Gustav Fischer ed.. Springer Verlag.

Whal.en N.M. and SChaTTE K.E.

1997 Pleistocene sites in southern Yemen. Arabian Archaeology and Epigraph: $8: 1-10$.

Wil.kinson T.J., Edens C. and Gibson M.

1997 The Archaeology of the Yemen High Plains: A preliminary chronology, Arabian Archaeology' and Epigraphy, 8 : 99-142. 